\title{
Fault Diagnosis for Industrial Images Using a Min-Max Modular Neural Network
}

\author{
Bin Huang and Bao-Liang Lu \\ Department of Computer Science and Engineering, Shanghai Jiao Tong University \\ 1954 Hua Shan Rd., Shanghai, P.R. China 200030 \\ uu.hb0@public1.sz.js.cn, blu@cs.sjtu.edu.cn
}

\begin{abstract}
This paper presents a new fault diagnosis method for industrial images based on a Min-Max Modular $\left(\mathrm{M}^{3}\right)$ neural network and a Gaussian Zero-Crossing (GZC) function. The most important advantage of the proposed method over existing approaches such as radial-basis function network and support vector machines is that our classifier has locally tuned response characteristics and the misclassification rate of faulty product images can be controlled as small as needed by turning two parameters of the GZC function while the correct rate can be influenced to some extend. The experimental results on a real-world fault diagnosis problem of industrial images indicate that the effectiveness of the proposed method.
\end{abstract}

\section{Introduction}

The aim of developing a fault diagnosis system for industrial images described in this paper is to exactly classify all the CCD sensing images acquired from a product line into two categories: qualified product and faulty product. A strict requirement for the classifier used in fault diagnosis systems is that the classifier is not allowed to mistakenly classify images of faulty products into the category of qualified products. Although various image classification methods have been developed in the last few years [3], many of these methods, however, are not suitable to fault diagnosis for industrial images because they lack locally tuned response characteristics and the misclassification rate on faulty product images can not be controlled by a user.

In our previous work we have proposed a min-max modular $\left(\mathrm{M}^{3}\right)$ neural network [1] for dealing with large-scale pattern classification problem. As a kind of divide-and-conquer technique, the main idea of $\mathrm{M}^{3}$ network is to divide a complex multi-class problem into a series of smaller and simpler two-class subproblems and combine the individual solutions of all the subproblems into a solution to the original problem. An attractive feature of the $\mathrm{M}^{3}$ networks is that any multi-class problem can be decomposed into a number of linearly separable subproblems.

For linearly separable problems, we have proposed a Gaussian Zero-Crossing (GZC) function. The combination of the $\mathrm{M}^{3}$ network and the GZC function has two important advantages over existing classifiers. One is that fast learning 
without error can be achieved to any complex pattern classification problems; the other is that the locally tuned response features of the GZC function are maintained in the entire $\mathrm{M}^{3}$ network. In this paper, we apply the $\mathrm{M}^{3}$ network with the GZC function to a fault diagnosis problem of industrial images.

\section{Feature Extraction with Wavelet Transform}

\subsection{Image Processing}

A real-world image set used in this paper consists of CCD sensing images acquired from a product line at a leading manufacturing company. In this paper, all the images are in the form of 256 gray-scales bitmap and are classified into two classes: correct image and faulty image. The size of the images is $128^{*} 128$ in pixels. Two kinds of enlarged images are illustrated in Fig. 1, in which (a), (b) belong to correct image and (c) is classified into faulty image.

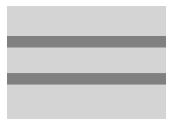

(a)

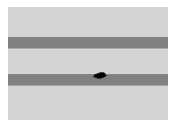

(b)

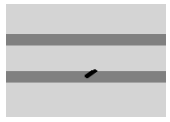

(c)

Fig. 1. Industrial CCD sensing images for qualified products (a) and (b), and defective product (c)

\subsection{Feature Extraction Using Wavelet Transform}

We used wavelet transform techniques to extract the features of images. The image data were convolved by using the Daubechies wavelets [6], which is orthogonal and with compact support. In the experiments below, the order $N$ was set to 2 with the encoding level 1 . After wavelet transforming, the dimension of the feature vector representing an image is 4225 .

\section{$3 \quad \mathrm{M}^{3}$ Network and GZC Function}

\section{$3.1 \quad \mathrm{M}^{3}$ Network}

The proposed method is based on a min-max modular neural network model [1]. With the task decomposition method, a $K$-class problem can be uniquely partitioned into a number of linearly separable problems, each of which includes only two different training data that belong to two classes. The number of linearly separable problems is given by

$$
\sum_{i=1}^{K-1} \sum_{j=i+1}^{K} L_{i} \cdot L_{j}
$$

where $L_{i}$ denotes the number of training data belonging to class $C_{i}$. 
After training individual component modules assigned to learn associated two-class sub-problems, all the trained component modules are integrated into a min-max modular neural network with the MAX, MIN, or/and INV units according to two module combination laws [1].

\subsection{Gaussian Zero-Crossing Discriminant Function}

A fatal weakness of linear discriminant functions is that they lack locally tuned response characteristics [2]. This deficiency may lead classifiers to mistakenly produce proper output even when an unknown input is presented.

To overcome the deficiency of linear discriminant functions, we have proposed a Gaussian Zero-Crossing (GZC) function for solving linearly separable problems in our previous work [2]. The definition of the Gaussian zero-crossing discriminant function is given by:

$$
f_{i j}(x)=\exp \left[-\left(\frac{\left\|x-c_{i}\right\|}{\sigma}\right)^{2}\right]-\exp \left[-\left(\frac{\left\|x-c_{j}\right\|}{\sigma}\right)^{2}\right]
$$

where $x \in \mathbf{R}^{n}$ is the input vector, $c_{i} \in \mathbf{R}^{n}$ and $c_{j} \in \mathbf{R}^{n}$ are the given training inputs belonging to class $\mathcal{C}_{i}$ and class $\mathcal{C}_{j}(i \neq j)$, respectively, and are used as two different receptive field centers, $\sigma=\lambda\left\|c_{j}-c_{i}\right\|$ is the receptive field width, $\lambda$ is a user-defined constant $(0<\lambda)$, and the norm $\|z\|$ is the Euclidean norm of vector $z$ [2]. An important advantage of the GZC discriminant function over existing linear discriminant functions is its locally tuned response characteristics.

The output for the $\mathrm{M}^{3}$ network with GZC discriminant function is defined by

$$
g(x)=\left\{\begin{array}{c}
1 \text { if } y_{i}(x)>\theta^{+} \\
\text {Unknown if } \theta^{-} \leq y_{i}(x) \leq \theta^{+} \\
-1 \text { if } y_{i}(x)<\theta^{-}
\end{array}\right.
$$

where $\theta^{+}$and $\theta^{-}$are the upper and lower threshold limits of the $\mathrm{M}^{3}$ network, respectively; and $y_{i}(x)$ denotes the transfer function of the $\mathrm{M}^{3}$ network for class $C_{i}$.

One attractive feature of the GZC discriminant function is that the proper generalization performance of the $\mathrm{M}^{3}$ network can be easily controlled by tuning $\theta^{+}$and $\theta^{-}$. From (2), we see that the receptive field width of the GZC function is determined by two factors: the constant $\lambda$ defined by a user and the distance between two different receptive field centers.

\section{Experiment Results}

To evaluate the effectiveness of the proposed method and compare it with support vector machines, we carry out simulations on a real-world fault diagnosis problem of industrial images. The training data set consists of 500 images and the test data set contains 83 images. Table 1 shows the distributions of the training and test data. 
Table 1. Distributions of The Training and Test Data

\begin{tabular}{l|c|c}
\hline \hline \multirow{2}{c|}{ Class } & \multicolumn{2}{|c}{ No. Data } \\
\cline { 2 - 3 } & Training & Test \\
\hline Images for qualified products & 400 & 100 \\
\hline Images for faulty products & 6 & 23 \\
\hline \hline
\end{tabular}

Table 2. Performance Comparison of $\mathrm{M}^{3}-\mathrm{GZC}$ with SVMs

\begin{tabular}{c|c|c|c|c|c}
\hline \hline & & \multicolumn{2}{|l}{ Success Rate $(\%)$} & \multicolumn{2}{c}{ Error No. in Testing } \\
\cline { 3 - 6 } Methods & SVs & Training & Test & Correct $\rightarrow$ Fault & Fault $\rightarrow$ Correct $^{b}$ \\
\hline $\mathrm{M}^{3}-$ GZC & N/A & 100.00 & 96.75 & 0 & 4 \\
\hline SVMs $(\sigma=2.0)$ & 137 & 100.00 & 96.75 & 0 & 4 \\
\hline SVMs $(\sigma=1.4)$ & 206 & 100.00 & 96.75 & 0 & 4 \\
\hline SVMs $(\sigma=1.0)$ & 328 & 100.00 & 92.69 & 0 & 9 \\
\hline SVMs $(\sigma=0.7)$ & 438 & 100.00 & 83.74 & 0 & 20 \\
\hline SVMs $(\sigma=0.5)$ & 460 & 100.00 & 81.30 & 0 & 23 \\
\hline \hline
\end{tabular}

$\bar{a}$ The number that the Correct are misclassified to the Fault.

${ }^{b}$ The number that the Fault are misclassified to the Correct.

\subsection{Experiment 1}

In this experiment, we compare our method with standard SVMs. Both $\theta^{+}$and $\theta^{-}$were set to 0.01 for the $\mathrm{M}^{3}$ network with the GZC function and $C$ for SVMs [4] was selected as $4,8,16,32$. The experimental results are shown in Table 2.

From Table 2, we see that our method has the same recognition rate on the whole test data as the standard SVMs. The results demonstrate that the $\mathrm{M}^{3}$ neural network with the GZC function has a well-performed structure for classification problems. When the radius $(\sigma)$ of the kernel function ${ }^{1}$ in SVMs is narrowed, however, the corresponding recognition rate become worse, especially, more number of images for defective products are mistakenly classified as the category of qualified products. From this result, we can point out that it is difficult for SVMs to reduce the misclassification rate on faulty images by shrinking the radius of the kernel function.

\subsection{Experiment 2}

In this experiment, we demonstrate that the rate of misclassifying the images of faulty products to the category of qualified products can be reduced to 0 with the use of the GZC discriminant function, while the correct recognition rate can be maintained in certain level. The results of the experiment is illustrated in Fig. 2. Here, $\theta^{-}=-\theta^{+}$. The unknown recognition rate means the percentage of the $M^{3}$ network producing 'I don't know' outputs. From Fig.2 (a) and (b), we can see that when $|\theta|$ is greater than or equal to 0.6147 , the error recognition

\footnotetext{
${ }^{1} \exp \left(\frac{\left\|X-X_{i}\right\|^{2}}{-2 \sigma^{2}}\right)$.
} 


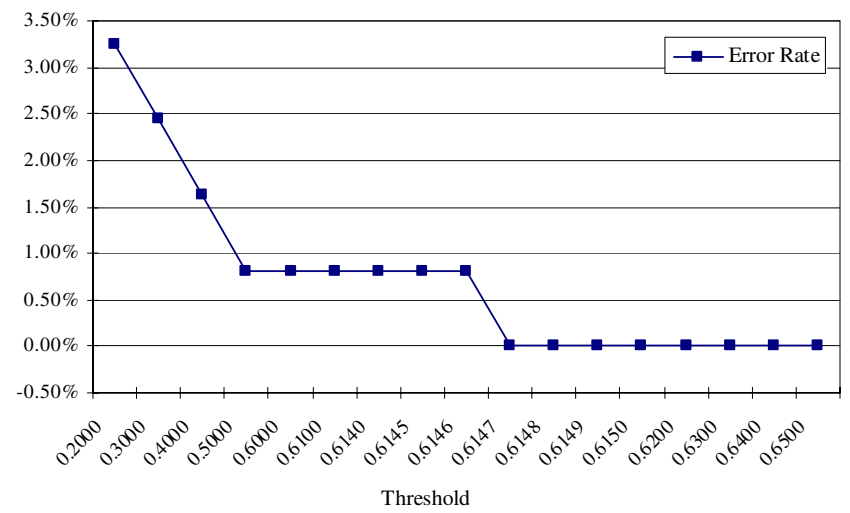

(a)

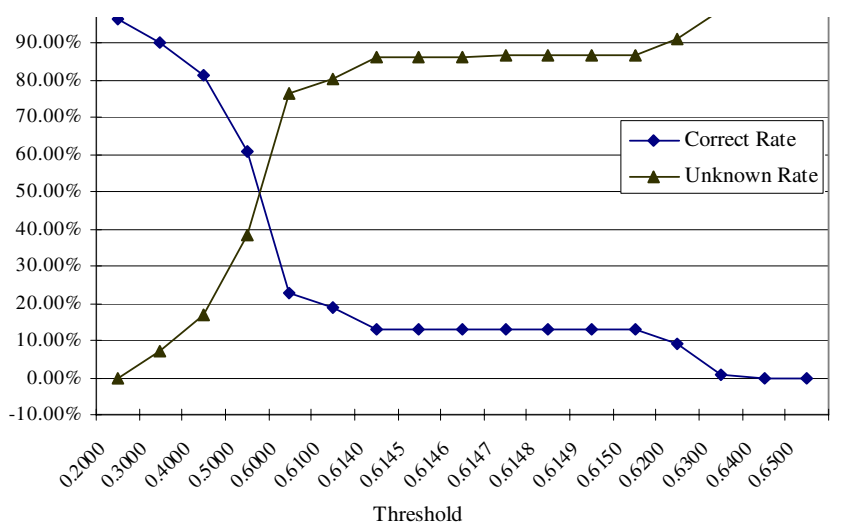

(b)

Fig. 2. Correct recognition rate and unknown recognition rate (a), and error recognition rate (b), as a function of the value of threshold of the $\mathrm{M}^{3}$ network

rate is reduced to $0 \%$. Meanwhile, the correct recognition rate is kept at $13.01 \%$. Although this correct recognitin rate is highly unsatisfactory for practical applications, the proposed method provides us with a promising approach to dealing with fault diagnosis problems of industrial images.

\section{Conclusions}

We have presented a fault diagnosis method based on the combination of the $\mathrm{M}^{3}$ neural network and the Gaussian Zero-Crossing discriminant function. The experiment results indicate that the proposed method have the advantage to reduce the misclassification rate on faulty products that SVMs are lack of in our experiments. As to future work, we will refine the internal classification structure for improving correct recognition rate while reducing the rate of misclassifying faulty product images. 


\section{Acknowledgements}

The authors would like to thank Mr. Kai-An Wang for the help on simulations. This work was partially supported by the National Natural Science Foundation of China via the grant NSFC 60375022.

\section{References}

1. Lu, B.L., Ito, M.: Task Decomposition and Module Combination Based on Class Relations: A Modular Neural Network for Pattern Classification. IEEE Trans. on Neural Networks, Vol. 10 (1999) 1244-1256

2. Lu, B.L., Ichikawa, M.: A Gaussian Zero-Crossing Discriminant Function for Min-Max Modular Neural Networks. Proc. of $5^{\text {th }}$ International Conference on Knowledge-Based Intelligent Information Engineering Systems \& Allied Technologies, (2001) 298-302

3. Barla, A., Odone, F., Verri, A.: Old Fashioned State-of-the-art Image Classification. Proceedings of $12^{\text {th }}$ International Conference on Image Analysis and Processing (2003) 566-571

4. Cristianini, N., Taylor, J.S.: An Introduction to Support Vector Machines and Other Kernel-based Learning Methods. Cambridge University Press (2000)

5. Richard O. Duda, R.O., Hart, P.E., Stock, D.G.: Pattern Classification (2nd Ed.). John Wiley \& Sons, Inc. (2001)

6. Daubechies, I.: Ten Lectures on Wavelets. SIAM (1992)

7. Hayashi, S., Asakura, T., Zhang, S.: Study of Machine Fault Diagnosis System Using Neural Networks. Proc. of IJCNN '02, Vol.1 (2002) 956 - 961

8. Asakura, T., Kobayashi, T., Xu, B.J., Hayashi, S.: Fault Diagnosis System for Machines Using Neural Networks. Trans. JSME, Vol.43, No.2 (2000) 364-372 